\section{Alfa-2a recombinant interferon in HIV associated thrombocytopenia}

We report a case of thrombocytopenia associated with human immunodeficiency virus (HIV) infection that was treated with interferon.

\section{Case report}

A male homosexual (aged 30) presented with bruising and gingival bleeding. His platelet count was $5 \times 10^{9} / 1$ and his bone marrow contained increased numbers of megakaryocytes. An impalpable spleen and absence of antinuclear-facto supported a diagnosis of immune thrombocytopenia. Prednisolone $60 \mathrm{mg}$ daily raised the platelet count to $66 \times 10^{9} /$, but he became jaundiced with "hepatitic" liver function values. He had been a low grade hepatitis B carrier (hepatitis B surface antigen titre on radioimmunoassay $6 \cdot 25$, $e$ antigen negative, and core antigen IgM equivocal) and was positive for HIV antibodies by Western blotting. After six weeks of prednisolone the hepatitis B surface antigen titre had risen to 134 (haemagglutination $>8192$ ) and he became positive for $e$ antigen and core antigen IgM, findings consistent with reactivation of hepatitis B. Steroids were discontinued. The platelet count fell but responded $\left(250 \times 10^{9} / 1\right)$ to intravenous immunoglobulin $0.4 \mathrm{~g} / \mathrm{kg}$ daily for five days. The effect was short lived and he required repeated intravenous immunoglobulin for a platelet count of less than $35 \times 10^{9} / 1$. After mild trauma he experienced right loin pain and profuse haematuria, indicating renal haemorrhage, which was managed by platelet infusions and intravenous immunoglobulin. Liver biopsy at six months showed chronic active hepatitis.

Recombinant alfa-2a interferon, $3 \mathrm{MU}$ on alternate days, was given subcutaneously. Core antigen IgM fell to equivocal values, e antigen became negative, and $\mathrm{e}$ antibody appeared. Liver function values became near normal. The platelet count, measured weekly, rose from 62 (SD 98) $\times 10^{9} / 1$ to 110 (49) $\times 10^{9} / 1$; the number of weeks the platelet count was below $35 \times 10^{9} / 1$ fell from 10 to 0 and the requirement for intravenous immunoglobulin fell from 15 days to four days over this period, being given when the platelet count appeared as if it might reach less than $35 \times 10^{9} / 1$ (figure). Interferon was continued for five months and then stopped; the platelet count fell to less than $35 \times 10^{9} / 1$ and further intravenous immunoglobulin was required. Interferon was restarted after three months. The platelet count rose again, and, although the rise in the mean weekly count was not as pronounced $\left(69(27) \times 10^{9} / 1\right)$, he did not require any further intravenous immunoglobulin over the four months, and the count did not fall below $35 \times 10^{9} /$.

Platelet associated immunoglobulin was measured before we started the second course of interferon and during interferon treatment using a fluorescence activated cell cytometer (Epics C, Coulter Electronics). Platelets obtained by centrifugation from blood stored in edetic acid and washed in phosphate buffered saline were then fixed by $1 \%$ paraformaldehyde to prevent in vitro adsorption of immune complexes. They were then reacted with fluorescein isothionate conjugated antihuman IgG and IgM. Platelet associated immunoglobulin was calculated as \% fluorescence using a standard curve. At the start of the second course of interferon, when the platelet count was $28 \times 10^{\%} /$, concentrations of both platelet associated IgG and IgM were raised $\left(25 \mathrm{ng} / 10^{6}\right.$ platelets (normal $<4.85$ ) and $15 \mathrm{ng} / 10^{6}$ platelets (normal $<1$ respectively). These concentrations fell with the platelet response. Immune complexes were not detected on the platelets or in serum.

\section{Comment}

This case illustrates that thrombocytopenia related to HIV infection is not always benign. ${ }^{1}$ The cytopenia is thought to reflect an intact immune

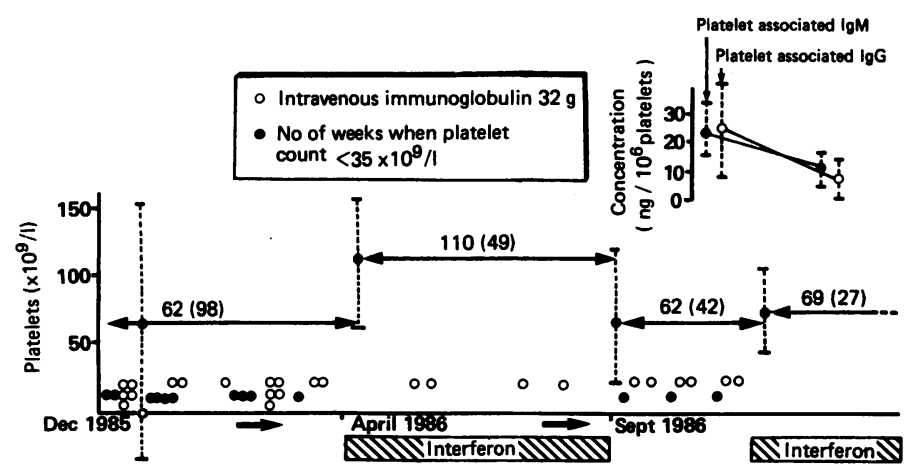

Mean (SD) platelet count and platelet associated immunoglobulin concentrations in relation to interferon treatment. response to HIV, occurring in up to $25-38 \%{ }^{2}$ of cases and resolving on progression to the full acquired immune deficiency syndrome (AIDS). The thrombocytopenia may be due to platelet autoantibody production ${ }^{3}$ or to viral related stem cell damage. ${ }^{4}$ Treatment is not always indicated unless there is bleeding or the count is very low. Treatment with steroids or splenectomy can precipitate Kaposi's sarcoma, opportunistic infections, and metabolic effects, and intravenous immunoglobulin is increasingly being considered as first line treatment.

This man developed chronic active hepatitis, which supports the notion that immunosuppressive manoeuvres should be avoided. The very high levels of platelet associated antibodies, particularly IgM, were at variance with the generally accepted mechanism of circulating immune complexes ${ }^{5}$ in homosexual associated idiopathic thrombocytopenia but agreed with the findings of others ${ }^{4}$ and confirmed a failure of control of the humoral immune system. The immune modulatory and anti-HIV activities of interferon might have been expected to exert an effect in such a patient. The use of interferon in our patient was indeed associated with an improvement in the platelet count, obviated the need for steroids, reduced the requirement for intravenous immunoglobulin, and deferred splenectomy. The reduction in platelet associated immunoglobulin in association with its use supports a causal effect. This approach requires further investigation.

We thank Miss M Macey, research fellow at The London Hospital, for her expert help with the platelet antibody measurements.

1 Abrams DI, Kiprov DD, Goedert JJ, et al. Antibodies to human T lymphocyte virus type III and development of the acquired immuno-deficiency syndrome in homosexual men presenting with immuno thrombocytopenia. Ann Intern Med 1986;104:47-50.

2 Treacy M, Lai L, Costello C, Clark A. Peripheral blood and bone marrow abnormality in patients with HIV related disease. Br $\mathcal{H}$ Haematol 1987;65:289-94.

3 Stricker RB, Abrams DO, Corash L, Shuman MA. Target platelet antigen in homosexual men with immuno thrombocytopenia. N Engl f Med 1985;313:1375-80.

4 Donahue RE, Johnson MM, Zon LI, Clark SC, Groupman JE. Suppression of in vitro haematopoiesis following human immunodeficiency virus infection. Nature 1987;326:200-3. 5 Karpatkin S. Auto-immune thrombocytopenic purpura. Seminars in Haematology 1985;22:260-88. (Accepted 28 October 1987).

Regional Department of Infectious Diseases and Tropical Medicine, Monsal Hospital, Manchester M10 8WR

M E ELLIS, MRCP, DCH, consultant physician

KR NEAL, MRCP, registrar

C L S LEEN, MRCP, senior registrar

The London Hospital, Whitechapel, London E1 1EB

A C NEWLAND, MRCP, MRCPATH, senior lecturer in haematology

Correspondence to: Dr Ellis.

\section{Treatment of thrombocytopenia with alfa interferon}

Idiopathic thrombocytopenia has traditionally been treated with steroids ${ }^{1}$ and immunosuppressive drugs ${ }^{2}$ and, if these fail, by splenectomy. Recently high dose intravenous gammaglobulins have also been used successfully, particularly in children. ${ }^{34}$

Human immunodeficiency virus (HIV) infection is associated with thrombocytopenia, ${ }^{5}$ which may be autoimmune. All the established treatments used in idiopathic thrombocytopenia have disadvantages in patients with HIV infection. Steroids and immunosuppressants may predispose to further infection, and splenectomy carries the risks of surgery. While not immunosuppressive, treatment with intravenous gammaglobulin usually requires admission to hospital and is expensive.

We describe two patients with unrelated causes of thrombocytopenia who received alfa (lymphoblastoid) interferon as part of their treatment for another disease and whose clinical and laboratory features of thrombocytopenia improved.

\section{Case 1}

A boy of Indian parents, born in 1974, was well until 1984, when easy bruising and epistaxes were noted. His platelet count was consistently below $30 \times 10^{9} / 1$ and his bone marrow showed plentiful megakaryocytes. Autoimmune thrombocyto- 